\title{
Some Techniques for Measuring Small Mutual Inductances
}

\author{
D. N. Homan*
}

\author{
Institute for Basic Standards, National Bureau of Standards, Washington, D.C. 20234
}

(July 6, 1966)

\begin{abstract}
A method of measuring small mutual inductances is presented. The smallest inductor measured was $0.1 \mu \mathrm{H}$. The circuit is a transformer-ratio-arm bridge with multiple balances and is described in detail. Uncertainties are of the order of one part in $10^{7}$.

Several ideas for the design and construction of suitable mutual inductance standards are presented.
\end{abstract}

Key Words: Auxiliary generator, bridge, coaxial choke, mutual inductor, stray magnetic field, transformer.

\section{Introduction}

A recent theoretical study by Page $[1]^{1}$ suggests the desirability of making accurate measurements of mutual inductance at the $0.1 \mu \mathrm{H}$ level and of stepping up to larger values. An experimental bridge has been constructed for the purpose of investigating some techniques which seemed appropriate in this range. Some of those techniques which appear to be particularly useful are described in this paper. The bridge is a transformer-ratio-arm bridge, having a ratio of 10 to 1 , and has been designed to compare mutual inductors through five orders of magnitude, from 0.1 $\mu \mathrm{H}$ to $1 \mathrm{mH}$ at a frequency of $1592 \mathrm{~Hz}$. Sensitivity of a part in $10^{7}$ is achieved at the $0.1 \mu \mathrm{H}$ to $1 \mu \mathrm{H}$ level provided the inductors carry $3 \mathrm{~A}$ in their primary windings.

\section{Bridge Circuit}

A simplified version of the bridge is shown in figure 1 . Mutual impedances $Z_{1}$ and $Z_{2}$, which are both mainly inductive, are defined by the equations

$$
\begin{aligned}
& Z_{1}=j \omega M_{1}+R_{1} \\
& Z_{2}=j \omega M_{2}+R_{2} .
\end{aligned}
$$

If both inductors have the same current, $i$, in their primary windings, ${ }^{2}$ the comparison of $Z_{1}$ to $Z_{2}$ is achieved by measuring the ratio of the induced voltages $e_{1}$ and $e_{2}$. Since $Z_{1}=\frac{e_{1}}{i}$ and $Z_{2}=\frac{e_{2}}{i}$, then $\frac{Z_{1}}{Z_{2}}=\frac{e_{1}}{e_{2}}$. The ratio of $e_{1} / e_{2}$ is compared with the 10 to 1 voltage ratio of an inductive voltage divider, which may be calibrated by a modification of the capacitance-ratio

*Present address: High Frequency Impedance Standards Section. NBS. Boulder, Colo. 80302

${ }^{1}$ Figures in brackets indicate the literature references at the end of this paper.

${ }^{2}$ Ground capacitance current between the primary windings is negligible; it is discussed in section 4 of this paper. method described by Cutkosky and Shields [2]. The bridge balance is accomplished by the adjustment of $e_{3}$, which is an adjustable voltage source, small relative to $e_{1}$ and $e_{2}$.

The detector consists of a tunable amplifier and a tuned high-impedance preamplifier, coupled to the bridge through an impedance matching transformer. The transformer is tuned with a variable capacitor to give maximum output voltage.

Accurate measurement of small inductances requires that all sources of stray magnetic field be well controlled. To that end, inductors and transformers are toroidal, bridge components are enclosed in copper eddy-current shields, components are interconnected by coaxial cables, and shield current of the coaxial leads is restricted to be nearly equal and opposite to current in their inner conductors. Figure 2 illustrates shielding and the use of the technique of threading a coaxial lead through a high permeability core [3] forming a coaxial choke to equalize current in the inner conductor and outer shield of the coaxial cable.

Following construction of the bridge, tests were made to establish the effectiveness of the design in keeping stray magnetic fields small. Such tests were made by using two coils of wire, one connected to a

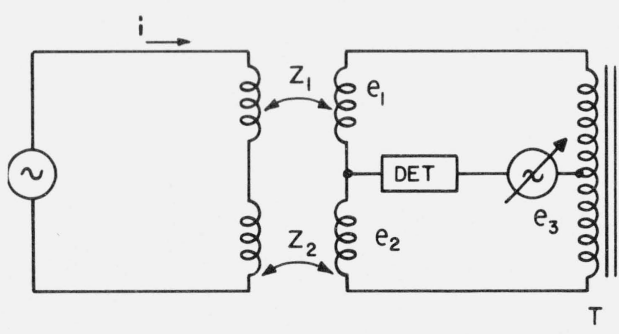

FigURE 1. Simplified inductance bridge circuit. 


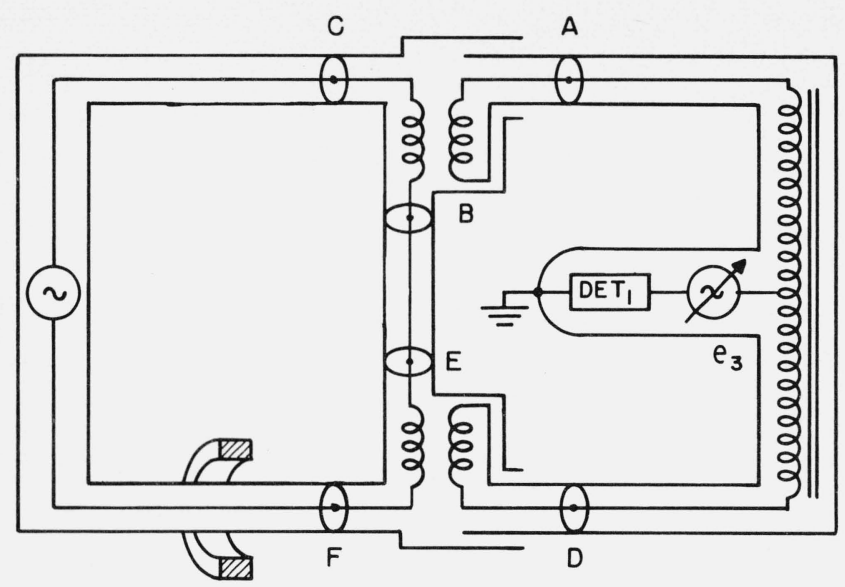

FIGURE 2. Inductance showing shielding, junction points $(A, B$, $C, D, E, F)$ of the mutual inductor, and use of high-permeability core to equalize coaxial current.

detector and used to find sources of stray field, and the other connected to a generator and used to find places in the circuit that were sensitive to stray field. For example, the detector coil was moved around until a source of stray field was found, and the amount of detector off-balance was noted. The coil connected to the generator was driven so that its field gave about the same off-balance at a comparable distance from the detector coil. Then the driven coil was used as a probe to determine the effect of such a field on the main bridge balance when brought near various bridge components.

The largest sources of stray field were the coaxial leads in the loop that includes the inductor primary windings since the current is largest in that part of the circuit. It was determined that the coaxial choke, mentioned above, was not sufficient to equalize current in the inner conductor and outer shield of the coaxial cable. A greatly improved coaxial choke was realized by making it an active device using two cores in a manner described by Gibbings [4]. As it is used in this application, it may be called an "active coaxial choke." The principle is shown in figure 3 . If $e_{P}=Z_{P} i, e_{Q}=G e_{P}$, and $e_{T}=e_{P}+e_{Q}$, it can be shown that $Z_{T}=Z_{P}(1+G)$. Thus, the active choke reduces net current much more than a coaxial choke composed of a single magnetic core. The gain of the amplifier and the number of turns of cable through the device was adjusted to reduce net current by an amount which made the error negligible.

Small stray magnetic fields due to slight nonsymmetries in the coaxial cables remained. Using the probe technique discussed above, three modifications were made. First, the copper can serving as an eddycurrent shield for the inductive divider was found to be unsatisfactory because the lid was electrically attached to the can at only one point. The shield was improved by bolting the lid to the can firmly with screws spaced about 2 in apart around its circum-

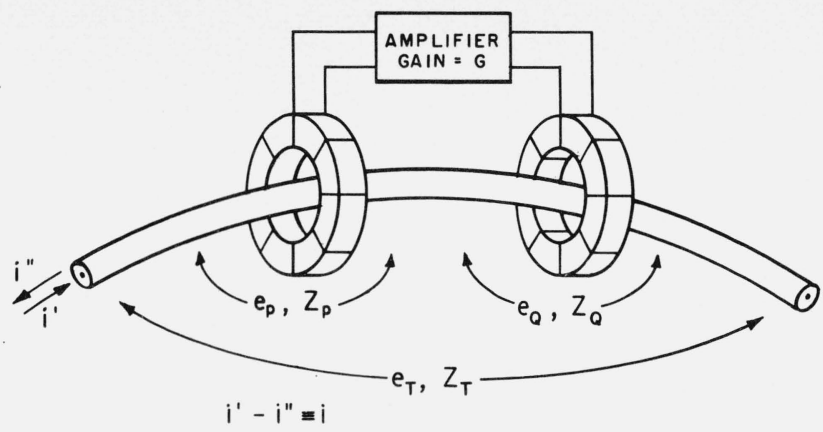

Figure 3. Active coaxial choke.

ference. Second, an uncertainty in the value of mutual inductance arises from the coupling between the leads connected to the inductor primary windings and the leads connected to the inductor secondary windings, if the leads move relative to each other. Subsequent inductors were designed to have the primary winding terminals on the opposite side of the shield can from the secondary terminals. Third, the smallest inductors (for which the ratio of mutual inductance of the leads to mutual inductance of the inductor is largest) were modified as follows: The eddy-current shield was extended over the secondary leads by passing these leads through a three-inch-long copper cylinder having $1 / 8$-in wall thickness (about two skin depths at the frequency used).

The standards to be compared, as illustrated in figure 2, are defined as follows: Let $i_{A}^{\prime}$ be the current into the inner terminal of connector $\mathrm{A}, i_{4}^{\prime \prime}$ be the current out of the outer terminal, and $e_{A}$ be the voltage from the inner to the outer terminal. Currents and voltages are similarly defined at connectors B, C, D, $\mathrm{E}$, and $\mathrm{F}$. Then the mutual impedances of interest are defined by $Z_{A B}=\frac{e_{A}}{i_{B}^{\prime}}$ with the conditions $i_{B}^{\prime}=i_{B}^{\prime \prime}, e_{B}$ $=0$, and $i_{A}^{\prime}=i_{A}^{\prime \prime}=0 ; \quad Z_{D E}=\frac{e_{D}}{i_{E}^{\prime}}$ with the conditions $i_{E}^{\prime}=i_{E}^{\prime \prime}, e_{E}=0$, and $i_{D}^{\prime}=i_{D}^{\prime \prime}=0$. The above conditions are similar to those used in the theoretical analysis by Cutkosky of four-terminal-pair networks [5].

\section{Auxiliary Balances}

The techniques used to assure the realization of the conditions defining the inductors involve the use of several auxiliary generators and detectors. Figure 4 shows the complete system. Each of the four auxiliary generators consists of a decade capacitance network in parallel with a decade conductance network, driven by a 40-turn winding on the magnetic core of the bridge supply transformer. Figure 4 shows two such 40-turn windings, the sign of the voltage of one being opposite to that of the other so that each capacitance decade or conductance decade can be switched 


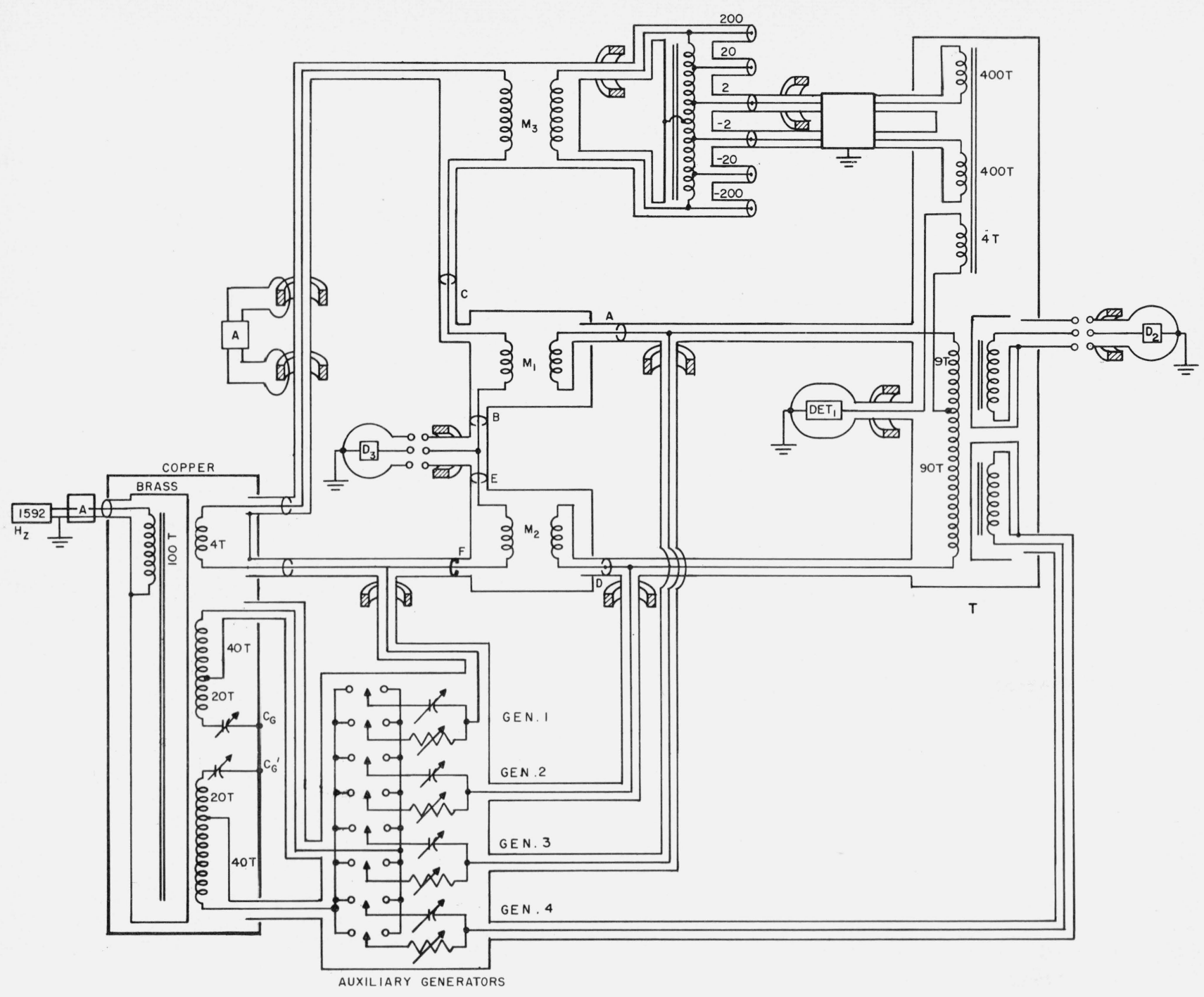

FiguRE 4. Complete system for measurement of small mutual inductances.

independently to reverse the sign of the voltage driving it. Stray capacitance from the 40 -turn winding to the shield raises the potential of the shield and would result in current in the shield. This difficulty is overcome if an adjustable capacitor, $C_{G}$, is attached to the 20-turn winding, the voltage of which is opposite in sign to that of the 40-turn winding. A capacitance bridge is formed by placing a detector between the shield and the bottom of the 40-turn winding, and the bridge can be balanced by adjusting $C_{G}$. A similar bridge is formed with $C_{G}^{\prime}$ as one component. Permanent adjustments of $C_{G}$ and $C_{G}^{\prime}$ were made following the completion of this component of the system.

The load on the secondary winding of each inductor is supplied by an auxiliary generator. The loading consists of capacitance in the cable connecting the inductors to the inductive voltage divider, $T$, and capacitance in the inductive voltage divider itself. Each of the auxiliary generators is adjusted until unplugging the inner conductor of the coaxial connection at the inductor secondary shows no change in the null at detector No. 1. Such a condition can be expressed as $i_{A}^{\prime}=0$ and $i_{D}^{\prime}=0$. The impedances of the auxiliary generators are high relative to the inductor secondary windings so that convergence to balance is more quickly achieved. Without the auxiliary generators, the loading in the circuit is such that the voltage $e_{A}$ at connector $\mathrm{A}$ of a $100 \mu \mathrm{H}$ inductor was changed by approximately one part in $10^{5}$ when the load was disconnected from the circuit by unplugging the lead to the secondary winding. Thus the auxiliary generators need to be adjusted to one part in $10^{3}$ to obtain accuracy of one part in $10^{8}$. 
At the $1 \mathrm{mH}$ level, an error arises from a capacitance change at the secondary terminal when a coaxial cable is connected to the terminal. When such a connection is made, the inner connector of the coaxial cable slips over the inner member of the connector on the inductor, thus changing the capacitance from the inner connector of the inductor to its shield about $0.3 \mathrm{pF}$. The impedance of the secondary winding of the $1 \mathrm{mH}$ inductor used in this work is about $20 \Omega$. A calculation shows that one could expect an error of about 6 in $10^{8}$ due to such a capacitance change. This error can be made reproducible by using the same coaxial cable each time the $1 \mathrm{mH}$ inductor is measured.

The inductive voltage divider T is a two-stage device. Two stage devices have been analyzed by Gibbings [4] and by Cutkosky [6]. The auxiliary generator (gen. 4 in fig. 4) incorporated in the transformer supplies the excitation current in the ratio winding. With the bridge balanced, a detector winding on the second core assures that no excitation current is supplied by the inductor secondary windings. Thus this inductive voltage divider behaves like a device having infinite input impedance in that it draws no current. An additional feature of the two-stage design is a highly accurate 10 to 1 ratio [4].

The conditions $e_{B}=0$ and $e_{E}=0$ are met by adjustment of the auxiliary generator 1 in the part of the circuit containing the primary windings of the mutual inductors such that detector No. 3 indicates null. Shunt admittances in conjunction with the impedance of the coaxial cable from the detector junction to points B or E may cause an error. A calculation based on an assumed equivalent circuit and approximate values for the impedance and admittance indicates that such an error is in the order of 1 in $10^{8}$ or less for typical length of coaxial cable used at this point. An experimental verification of the above calculation was made by observing the difference in the main bridge balance caused by changing the length of coaxial cable between $\mathrm{B}$ and $\mathrm{E}$.

Tests of interdependence of the detector balances were made by bringing the bridge to balance, and then changing one auxiliary generator voltage while observing changes in the null conditions at the detector junctions. Such tests and experience in bringing all the detectors to a simultaneous null indicate some dependence of one balance on another. However, the time involved in obtaining convergence of all balances is not unreasonably long.

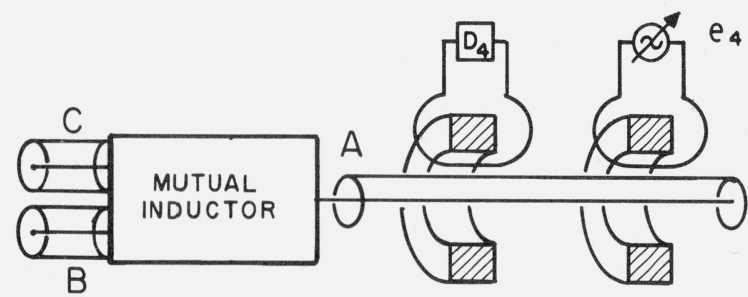

FIGURE 5. Method of assuring zero net current at inductor secondary terminal.
The condition $i_{A}^{\prime}=i_{A}^{\prime \prime}$ can be assured by an auxiliary device illustrated in figure 5 . One can adjust $e_{4}$ (to null $D_{4}$ ) to compensate for net current which can result from capacitance from the primary to the secondary winding of an inductor. However, it is possible to construct inductors in such a way that the change in the main bridge balance (detector 1 ) when $e_{4}$ is switched in and out is small enough so that the corresponding error is less than 1 part in $10^{8}$. The $1 \mathrm{mH}$ mutual inductor was altered by inserting a permanent short between the low-voltage side of the primary winding to the low-voltage side of the secondary. Inductors smaller than $1 \mathrm{mH}$ were not fixed with a short as described above since the error corresponding to the change in the main bridge balance when $e_{4}$ is switched in and out in their case was smaller than 1 in $10^{8}$ without the short.

\section{Main Bridge Balance}

The circuit used to obtain the small adjustable voltage, $e_{3}$ is shown in figure 6 . That portion of figure 6 shown within dotted lines represents a component part of an "a-c direct-reading-ratio set" which has been described by Cutkosky [6]. This is an active circuit with high input impedance and low output impedance. Hence $M_{3}$ is not strongly loaded, and reasonably large loads can be driven by the circuit. The in-phase and quadrature components of $e_{3}$ are read directly from dials of two inductive voltage dividers, IVD\# 1 and \# 2 in figure 6 . The mutual inductor $M_{3}$ has nearly the same current in its primary winding as the inductor $M_{1}$. The voltage $e_{3}$ is related, through current $i$, to $e_{1}$ and $e_{2}$. The relationship is controlled by the choice of the value of $M_{3}$, which provides a scale factor. The value of $M_{3}$ has been calculated such that one step of the last dial is one part in $10^{8}$.

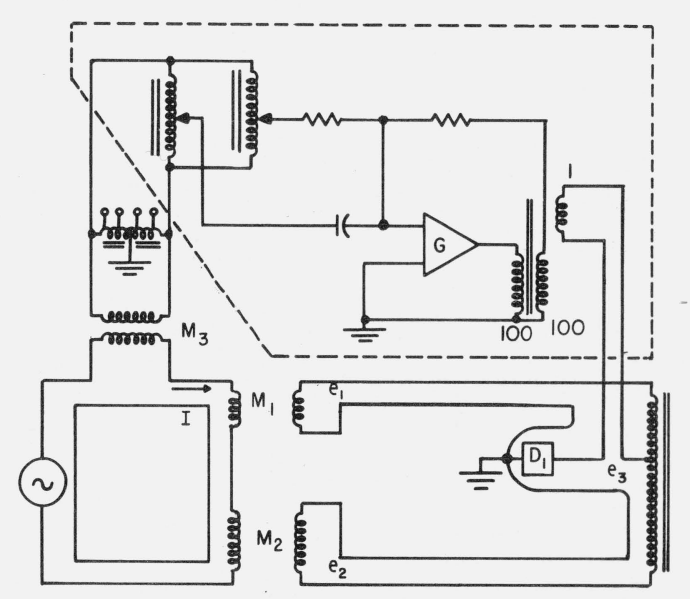

Figure 6. Basic bridge and, in dotted lines, circuit used to obtain balance. 
The range of $e_{3}$ is \pm 5 in $10^{4}$ and the least count is 1 in $10^{8}$. The current in $M_{3}$, therefore, must be no more than 2 in $10^{5}$ different from that in $M_{1}$ for accuracy of 1 in $10^{8}$. A calculation shows that ground capacitance between $M_{1}$ and $M_{3}$ and in $M_{3}$ must not exceed $1000 \mathrm{pF}$ for this accuracy. The inductor $M_{3}$ is constructed to have ground capacitance well below 1000 $\mathrm{pF}$. Components of the apparatus shown within dotted lines in figure 6 were initially adjusted to an accuracy of a few parts in $10^{5}$. Past experience with the type components used in the apparatus indicates that at the time of these measurements the accuracy of the dial readings was better than 1 part in $10^{3}$ of the dial reading. Thus if the inductors being compared (i.e., $M_{1}$ and $M_{2}$ ) are adjusted so that their values differ by less than 1 in $10^{5}$, the measurements can be relied upon to 1 in $10^{8}$.

\section{Measurement Procedure}

A single detector is used for all balances; it is plugged alternately into positions $D_{1}, D_{2}$, and $D_{3}$ (fig. 4). Balancing starts by bringing $D_{1}$ to null by the dials IVD\#1 and IVD\#2 (fig. 6). Next, leaving the detector at $D_{1}$, the secondaries of the mutual inductors $M_{1}$ and $M_{2}$ are unplugged in turn. With the secondary $M_{1}$ unplugged, a null at $D_{1}$ is obtained by turning the dials of gen. 3 (fig. 4); similarly, the secondary of $M_{2}$ is unplugged, and the balance reached with gen. 2 . The detector is then plugged into the $D_{2}$ position and a null is achieved by turning dials of gen. 4 . Finally, the detector is plugged into the $D_{3}$ position and brought to null by gen. 1 .

All balances described above are then repeated several times until convergence is reached.

\section{Inductors}

Some of the requirements of the inductors are that they be stable, astatic, have small phase angle, be close to nominal value, and be capable of carrying substantial current in their primary windings.

To make the inductors astatic, both the primary and secondary have toroidal symmetry about the same axis; the primary winding is outside the secondary winding and, therefore, has a larger cross section. The leads, as shown in figure 7 , are brought out to the

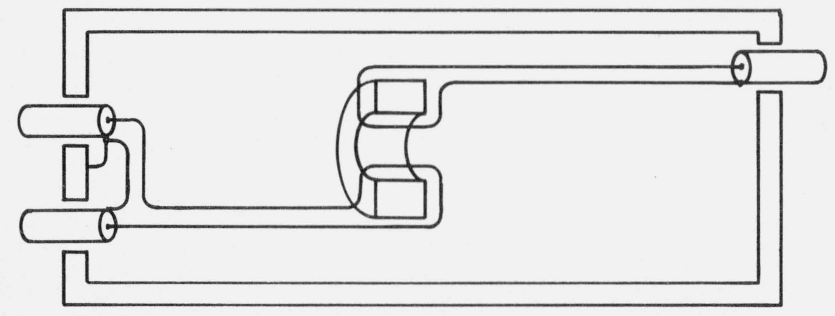

FIGURE 7. Lead geometry and shielding of mutual inductor. coaxial connectors in such a way as to create a minimum loop. The inductor is mounted in a copper shield of $1 / 8$-in thickness (about two skin depths at $1592 \mathrm{~Hz}$ ). The coaxial connector of one of the primary leads is connected to the copper shield at one point inside the shield. The primary leads and the secondary leads are brought out on opposite sides of the toroid as shown in figure 7 to keep magnetic coupling to a minimum outside the toroid.

If two windings have toroidal symmetry about a common axis, and if one of these windings is totally within the other, the mutual inductor thus formed has been observed to have the following property: The phase angle of the inductor is practically independent of the thickness of the wire used for the outside winding; the phase angle depends on the thickness of the wire used on the inside winding, a larger phase angle being produced by thicker wire. The outside winding is, therefore, better suited for the primary, since the primary should have low resistance for minimum heat dissipation.

A few inductors have been constructed using toroidal forms of Bakelite; they were potted in epoxy resin and enclosed in 1/8-in thick copper shield cans. These inductors are useful for testing some of the features of the inductance bridge, but have temperature coefficients that are too large to make them useful as standards in this work.

A $0.1 \mu \mathrm{H}$ inductor was made by painting silver windings onto a toroidal form of fused silica. The silica form, with the silver secondary winding, was baked and then a primary of copper wire was wound around the form but mechanically isolated from it. A critical problem is that of bringing the leads from the secondary out through the primary (see fig. 8). A cylindrical fused-silica rod was fused to the toroidal form as in figure 9 so that the leads could be brought out on a solid mount. An attempt to use silver painted leads was made but abandoned in favor of using \#36 copper wire. These lead wires were tightly twisted

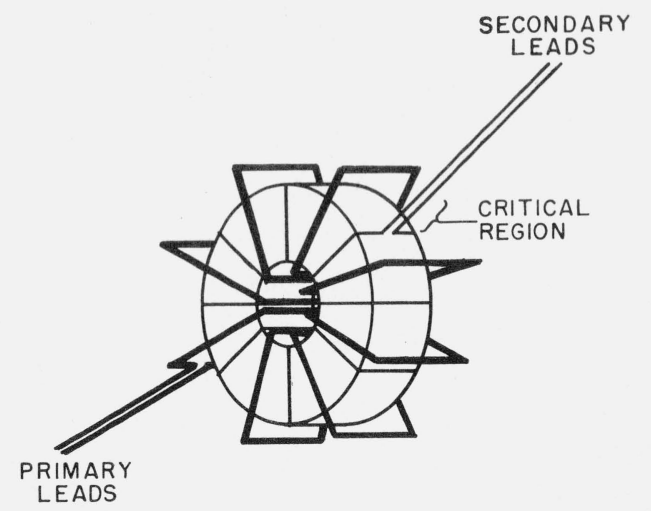

FIGURE 8. Winding geometry of mutual inductors having fused silica toroidal form. 


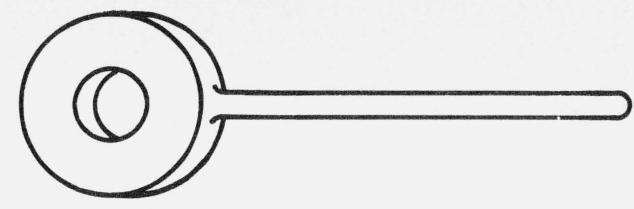

FigURE 9. Fused silica toroidal form and lead mount.

and attached to the fused-silica rod with alkyd resin paint, and electrical contact to the secondary was made with silver paint. The gage of the wire was chosen to be as small as possible to keep the loop area small, but large enough such that lead resistance was below the resistance of the painted secondary.

A possible source of uncertainty due to relative motion between primary and secondary is the magnetic coupling from the "single turn" produced by the progression of the turns about the toroid. This is an effective loop around the toroid in which an emf can be induced by a component of magnetic field parallel to the axis of the toroid. To reduce this uncertainty all the skew of the turns was concentrated at the inner surface of the toroid as shown in (fig. 10). After a thin insulating coating of alkyd resin paint was applied, a return loop was painted on top of the skewed wires forming a loop in the opposite direction to the loop formed by the skew.

A nonuniformity of the secondary winding painted on the toroidal form could create a net loop which would cause the inductors in effect to be lopsided. Thus relative motion between the primary and secondary windings could change the inductance. A calculation for the $0.1 \mu \mathrm{H}$ inductor indicates that such a net loop of $1 \mathrm{~mm}^{2}$ would dictate that for stability of one part in $10^{8}$, the relative motion of primary to secondary must be $10^{-5} \mathrm{~mm}$.

It appears that improved techniques in painting the secondary winding onto a fused-silica toroidal form would result in inductors of good stability.

(Paper 70C4-233)

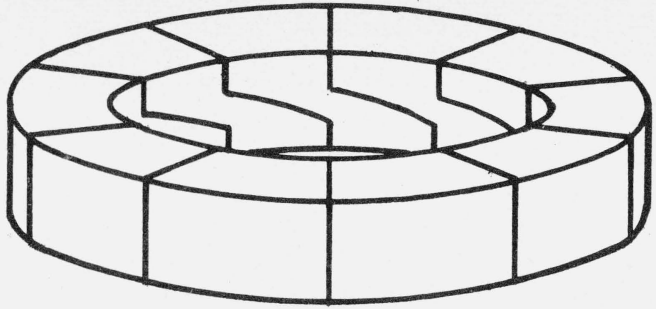

FigURE 10. Illustration of painted secondary winding showing skew of turns concentrated in inner toroidal surface.

\section{Conclusion}

At the smallest level of inductance measured here, bridge sensitivity is one part in $10^{7}$. Sensitivity improves at larger values of inductance such that $100 \mu \mathrm{H}$ can be compared with $1 \mathrm{mH}$ with sensitivity of a few parts in $10^{8}$. It appears likely that the bridge techniques described here can be relied on to yield errors that are less than one part in $10^{7}$. An analysis of all errors in such a step-up from $0.1 \mu \mathrm{H}$ to $1 \mathrm{mH}$ must await the development of a more reliable set of mutual inductance standards.

The author thanks R. D. Cutkosky for suggesting the scheme of measurement used here.

\section{References}

[1] C. H. Page, A new type of computable inductor, J. Res. NBS 67B (Math. and Math. Phys.) 1, (Jan.-Mar. 1963).

[2] R. D. Cutkosky and J. Q. Shields, The precision measurement of transformer ratios, IRE Trans. Inst. 1-9, 2 (1960).

[3] A. M. Thompson, The precise measurement of small capacitances, IRE Trans. Inst. 1-7, 3 and 4 (1958).

[4] D. L. H. Gibbings, A Circuit for Reducing the Exciting Current of Inductive Devices, The Proceedings of the Institution of Electrical Engineers, London, England, 108, Part B, 39 (1961).

[5] R. D. Cutkosky, Four-terminal-pair networks as precision admittance and impedance standards, IEEE Trans. Communications and Electronics, pp. 19-22 (Jan. 1964).

[6] R. D. Cutkosky, Active and passive direct reading ratio sets for the comparison of audio frequency admittances, J. Res. NBS 68C (Engr. and Instr.) (Oct.-Dec. 1964). 